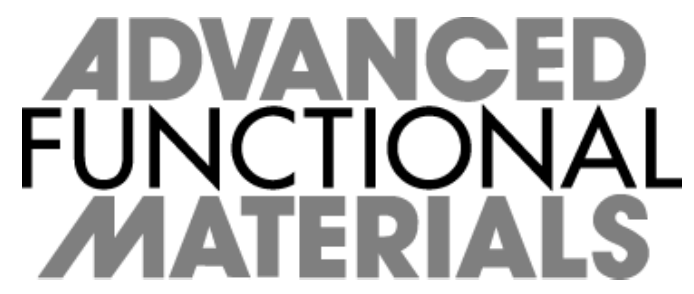

Dear Author,

Please correct your galley proofs carefully and return them no more than four days after the page proofs have been received.

Please limit corrections to errors already in the text; cost incurred for any further changes or additions will be charged to the author, unless such changes have been agreed upon by the editor.

The editors reserve the right to publish your article without your corrections if the proofs do not arrive in time.

Note that the author is liable for damages arising from incorrect statements, including misprints.

Please note any queries that require your attention. These are indicated with a $Q$ in the PDF and a question at the end of the document.

Reprints may be ordered by filling out the accompanying form.

Return the reprint order form by fax or by e-mail with the corrected proofs, to WileyVCH : afm@wiley.com
Postfach 101161 69451 Weinheim

Germany

WILEY-VCH
Courier services:

Boschstraße 12

69469 Weinheim

Germany

Tel.: (+49) 6201606531

Fax: (+49) 6201606500

E-mail: afm@wiley.com
To avoid commonly occurring errors, please ensure that the following important items are correct in your proofs (please note that once your article is published online, no further corrections can be made):

- Names of all authors present and spelled correctly

- Titles of authors correct (Prof. or Dr. only: please note, Prof. Dr. is not used in the journals)

- Addresses and postcodes correct

- E-mail address of corresponding author correct (current email address)

- Funding bodies included and grant numbers accurate

- Title of article OK

- All figures included

- Equations correct (symbols and sub/superscripts)

Corrections should be made directly in the PDF file using the PDF annotation tools. If you have questions about this, please contact the editorial office. The corrected PDF and any accompanying files should be uploaded to the journal's Editorial Manager site. 


\section{Author Query Form}

Dear Author,

During the copyediting of your manuscript the following queries arose.

Please refer to the query reference callout numbers in the page proofs and respond to each by marking the necessary comments using the PDF annotation tools.

Please remember illegible or unclear comments and corrections may delay publication.

Many thanks for your assistance.

\begin{tabular}{|c|l|l|}
\hline Query No. & \multicolumn{1}{|c|}{ Description } & \multicolumn{1}{|c|}{ Remarks } \\
\hline Q-OO & $\begin{array}{l}\text { Open access publication of this work is possible via Wiley OnlineOpen. Information about } \\
\text { this is available at: https://authorservices.wiley.com/author-resources/Journal-Authors/ } \\
\text { licensing-open-access/open-access/onlineopen.html. } \\
\text { The cost of publishing your manuscript OnlineOpen may be covered by one of Wiley's } \\
\text { national agreements. To find out more, visit https://authorservices.wiley.com/author- } \\
\text { resources/Journal-Authors/open-access/affiliation-policies-payments/index.html. } \\
\text { Note that eligibility for fee coverage is determined by the affiliation of the primary } \\
\text { corresponding author designated at submission. Please log in to your Wiley Author } \\
\text { Services account at https://authorservices.wiley.com/ and confirm your affiliation to see } \\
\text { if you are eligible. } \\
\text { Instructions for placing an OnlineOpen order can be found at: https://authorservices. } \\
\text { wiley.com/author-resources/Journal-Authors/open-access/how-to-order-onlineopen.html. }\end{array}$ & $\begin{array}{l}\text { To publish your article open access, please complete the order process before completing } \\
\text { your proof corrections. }\end{array}$ \\
\hline Q1 & $\begin{array}{l}\text { Please confirm that forenames/given names (blue) and surnames/family names } \\
\text { (vermilion) have been identified correctly. }\end{array}$ & \\
\hline Q2 & $\begin{array}{l}\text { Please provide the highest academic title (either Dr. or Prof.) for all authors, where } \\
\text { applicable. }\end{array}$ & \\
\hline Q4 & $\begin{array}{l}\text { Please shorten Abstract text to a maximum of 200 words. All abbreviations should be } \\
\text { defined. }\end{array}$ & $\begin{array}{l}\text { Please shorten Table of Contents text to a maximum of 60 words. All abbreviations should } \\
\text { be defined. }\end{array}$ \\
\hline
\end{tabular}

Please confirm that Funding Information has been identified correctly.

Please confirm that the funding sponsor list below was correctly extracted from your article: that it includes all funders and that the text has been matched to the correct FundRef Registry organization names. If a name was not found in the FundRef registry, it may not be the canonical name form, it may be a program name rather than an organization name, or it may be an organization not yet included in FundRef Registry. If you know of another name form or a parent organization name for a "not found" item on this list below, please share that information.

\begin{tabular}{|l|l|}
\hline FundRef Name & FundRef Organization Name \\
\hline Engineering and Physical Sciences Research Council & Engineering and Physical Sciences Research Council \\
\hline H2020 European Research Council & H2020 European Research Council \\
\hline UK Regenerative Medicine Platform & \\
\hline $\begin{array}{l}\text { UK Regenerative Medicine Platform Hub Acellular SMART materials } \\
\text { 3D architecture }\end{array}$ & \\
\hline UK Regenerative Medicine Platform & \\
\hline EPSRC & Engineering and Physical Sciences Research Council \\
\hline National Key Research and Development Program of China & National Key Research and Development Program of China \\
\hline National Natural Science Foundation of China & National Natural Science Foundation of China \\
\hline
\end{tabular}




\section{FULL PAPERS}

B. O. Okesola, S. Ni, B. Derkus,

C. C. Galeano, A. Hasan, Y. Wu,

J. Ramis, L. Buttery, J. Dawson, M. D'Este, R. OC Oreffo, D. Eglin,

H. Sun,* A. Mata*. 1906205

\section{Growth-Factor Free Multicomponent}

Nanocomposite Hydrogels That

Stimulate Bone Formation



A multicomponent hydrogel platform that combines oxidative coupling with 3 supramolecular coassembly to enable 4 tunability of physical, mechanical, and 5 biological properties desirable in bone tissue regeneration is reported. Specifi- 7 cally, the strategy permits the integration 8 of the osteogenic properties of Laponite (Lap), the nanofibrous structure of pep- 10 tide amphiphiles, the proangiogenic 11 properties glycyl-histidyl-lysine peptide, and the instant gelation properties of 13 tyramine-modified hyaluronic acid. 


\title{
Growth-Factor Free Multicomponent Nanocomposite Hydrogels That Stimulate Bone Formation
}

\author{
Babatunde O. Okesola, Shilei Ni, Burak Derkus, Carles C. Galeano, Abshar Hasan, \\ Yuanhao Wu, Jopeth Ramis, Lee Buttery, Jonathan Dawson, Matteo D'Este, \\ Richard OC Oreffo, David Eglin, Hongchen Sun,* and Alvaro Mata*
}

\section{Introduction}

Synthetic osteo-promoting materials that are able to stimulate and accelerate bone formation without the addition of exogenous cells or growth factors will represent a major opportunity for an aging world population. a coassembling system that integrates hyaluronic acid tyramine (HA-Tyr), bioactive peptide amphiphiles $\left(\mathrm{CHK}_{-} \mathrm{Cu}^{2+}\right)$, and Laponite (Lap) is reported to engineer hydrogels with physical, mechanical, and biomolecular signals that can be tuned to enhance bone regeneration. The central design element of the multicomponent hydrogels is the integration of self-assembly and enzyme-mediated oxidative coupling to optimize structure and mechanical properties in combination with the incorporation of an osteo- and angio-promoting segments to facilitate signaling. Spectroscopic techniques are used to confirm the interplay of orthogonal covalent and supramolecular interactions in the multicomponent hydrogels formation. Electron microscopy and analytical techniques are used to confirm coassembly by assessing changes in the nanostructures and elemental compositions of the multicomponent hydrogels. Furthermore, physicomechanical characterizations reveal that the multicomponent hydrogels exhibit improved compressive strength, stress relaxation profile, low swelling ratio, and retarded enzymatic degradation compared to the single component hydrogels. Applicability is validated in vitro using human mesenchymal stem cells and human umbilical vein endothelial cells, and in vivo using a rabbit maxillary sinus floor reconstruction model. Animals treated with the HA-TyrHA-Tyr-CHK-Cu${ }^{2+}$ hydrogels exhibit significantly enhanced bone formation relative to controls including the commercially available Bio-Oss.

There is an increasing need to develop 15 strategies to facilitate bone repair and 16 regeneration in and around missing or 17 defective craniomaxillofacial regions, 18 such as eyes, ears, noses, maxilla, man- 19 dible, and teeth. One area that requires 20 particular attention, given its anatomical 21 complexity and potential complications, is 22 the posterior maxillary. Here, loss of teeth 23 can result in adverse conditions including 24 severe maxillary sinus pneumatization, 25 which can also increase the risk of root 26 tips, teeth displacement into the sinus 27 cavity, or microbial contamination of the 28 maxillary sinus lift. ${ }^{[1]}$ Therefore, implan- 29 tation of osseointegrated biomaterials has 30 gained widespread attention in dentistry 31 to replace missing or lost teeth with suc- 32 cessful outcomes of complete or partial 33 edentulism. ${ }^{[2]}$ However, due to insufficient 34 alveolar bone and irregular structure of 35 the maxillary sinus in atrophied max- 36 illa, accidental displacement of a dental 37 implant into the maxillary sinus remains 38 a common complication encountered in 39 dental clinical practice. ${ }^{[3]}$ Consequently, 40 maxillary sinus floor reconstruction is 41

B. O. Okesola, B. Derkus, C. C. Galeano, A. Mata Institute of Bioengineering

Queen Mary University of London

London EI 4NS, UK

B. O. Okesola, B. Derkus, C. C. Galeano, A. Mata

School of Engineering and Materials Science

Queen Mary University of London

London E1 4NS, UK

S. Ni

Department of Oral Pathology

School and Hospital of Stomatology

Jilin University

Changchun 130000, China
B. Derkus

Biomedical Engineering Department

Faculty of Engineering

Eskisehir Osmangazi University

26040 Eskisehir, Turkey

A. Hasan, Y. Wu, A. Mata

School of Pharmacy

University of Nottingham

Nottingham NG7 2RD, UK

A. Hasan, Y. Wu, J. Ramis, L. Buttery, A. Mata

Biodiscovery Institute

University of Nottingham

Nottingham NG7 2RD, UK 
often necessary. Various bone-grafting biological materials including autologous grafts, allogenic bones, and xenografts are routinely used in the clinic to aid bone formation for the sinus floor. ${ }^{[4,5]}$ Notwithstanding, immunogenicity, donor site morbidity, disease transmission, scarcity of donors, and high cost are typically associated with these graft materials.

Significant research efforts have been expended to develop effective bone substitutes for maxillary sinus floor reconstruction. ${ }^{[6]}$ The majority of the studies have focused on the potency of bioceramics including hydroxyapatite, calcium phosphate cements, ${ }^{[7]}$ calcium sulfates, ${ }^{[8]}$ bioactive glasses, ${ }^{[9]}$ and calcium carbonates ${ }^{[10]}$ given their similarity with the inorganic components of natural bone and the ability of these materials to bind to bone and teeth. ${ }^{[11]}$ The success of this approach has resulted in a range of popular, commercially available, hydroxyapatite powders, cements, and granules including: Bio-Oss (Geistlich Ltd., Switzerland) and Fisiograft Bone (Ghimas S.p.A, Italy). In addition, hybridization of inorganic materials with polymers and/or growth factors has been explored as injectable composite materials for craniomaxillofacial bone tissue engineering. ${ }^{[12]}$ Similarly, membranes made from synthetic (exe. poly(L-lactic acid) (PLLA), polycaprolactone $(\mathrm{PCL}))^{[13,14]}$ or natural (exe. collagen, chitosan, alginate) ${ }^{[15,16]}$ polymers have been used to promote bone formation as well as peptides $^{[17]}$ or proteins ${ }^{[18,19]}$ to selectively stimulate relevant processes, such as mineralization. Nonetheless, an ideal bioactive scaffold for maxillary sinus floor reconstruction would enable the possibility to be delivered through minimally invasive means, while rapidly adapting to complex anatomical geometries. ${ }^{[20]}$

Hydrogels are attractive alternatives to bone grafts given their high water content, porosity for oxygen and nutrient permeability, biocompatibility, and responsiveness to environmental stimuli. Tailored-made hydrogels enable the incorporation of specific bioactive epitopes ${ }^{[21]}$ and exhibit physical properties, such as non-Newtonian behavior to facilitate injectability. ${ }^{[2]}$ However, the use of injectable hydrogels in maxillofacial surgery is limited and in most cases hydrogels have been used mainly as a delivery vehicle for growth factors, such as bone morphogenetic protein-2 (BMP-2) and vascular endothelial growth factor (VEGF). ${ }^{[23-25]}$ Furthermore, while growth factors are efficient

\section{J. Dawson, R. OC Oreffo}

Bone and Joint Research Group

Centre for Human Development

Stem Cells and Regeneration

Institute of Developmental Sciences

University of Southampton

Southampton SO16 6YD, UK

M. D'Este, D. Eglin

AO Research Institute Davos

Clavadelerstrasse 8, 7270 Davos Platz, Switzerland

H. Sun

Department of Oral and Maxillofacial Pathology

School and Hospital of Stomatology

China Medical University

Shenyang 110000 , China

E-mail: hcsun@jlu.edu.cn

A. Mata

Department of Chemical and Environmental Engineering

University of Nottingham

Nottingham NG7 2RD, UK

E-mail:a.mata@nottingham.ac.uk promoters of tissue growth, their use is associated with a variety 1 of critical complications, such as the need for large amounts of 2 growth factors due to the rapid inactivation and clearance of the 3 growth factors, ${ }^{[26]}$ high-costs, and uncontrolled activity. ${ }^{[27]} \quad 4$

Thus, an ideal therapeutic hydrogel for maxillary sinus lift 5 reconstruction/augmentation should also be biodegradable 6 generating nontoxic products, be simple to implant, exhibit 7 a short set time, be mechanically stable, and rapidly fill irreg- 8 ular anatomical volumes. ${ }^{[28]}$ Furthermore, given the complex 9 anatomy and physiology of bone, the hydrogel should hold 10 multifunctional properties to recreate key features of the extra- 11 cellular matrix (ECM) and stimulate cell types of interest. In 12 this context, multicomponent self-assembly offers an attractive 13 avenue to design hydrogels with multiple building-blocks, func- 14 tionalities, and the molecular precision of self-assembly. ${ }^{[29,30]} 15$

In this study, we have developed a three-component self- 16 assembling system that integrates hyaluronic acid (HA), pep- 17 tide amphiphiles (PAs), and Laponite (Lap). HA is a large ECM 18 polysaccharide ubiquitous in tissues and organs that has been 19 extensively used as a biomaterial due to its biocompatibility 20 and biodegradability. ${ }^{[31]}$ However, HA exhibits poor structural 21 integrity and stability and consequently is usually chemically 22 modified with, for example, tyramine $(\mathrm{Tyr})^{[32]}$ or hybridized 23 with other biomaterials, such as hydroxyapatite ${ }^{[33]}$ to increase 24 its functionality. PAs are a class of self-assembling peptide- 25 based building blocks with the intrinsic capacity to assemble 26 into well-defined nanofibrous hydrogels. ${ }^{[34]}$ PAs consist of: i) a 27 hydrophobic tail that drives self-assembly, ii) a $\beta$-sheet forming 28 amino acid sequence that stabilizes the assembled nanofibers 29 through hydrogen bonds, and iii) a charged functional head 30 group that facilitates solubility in aqueous environments. This 31 platform can incorporate a spectrum of bioactive epitopes, 32 which have been used to target regeneration of tissues, such 33 as bone, ${ }^{[35]}$ enamel, ${ }^{[36]}$ cartilage, ${ }^{[37]}$ and vascular. ${ }^{[38]}$ However, 34 self-assembling materials typically provide limited structural 35 integrity, which has hindered their wide spread applicability. 36 Lap is a 2D nanosilicate with anisotropic charge distribution, 37 which has been exploited as an effective cross-linker and rhe- 38 ology modifier for hydrogels ${ }^{[39]}$ for the delivery of drugs, growth 39 factors, and antibodies. ${ }^{[40,41]}$ Lap has been reported to promote 40 cell adhesion and proliferation and can exert osteogenic effects 41 on cells in vitro. ${ }^{[42]}$ Consequently, Lap has been combined with 42 macromolecules, such as $\mathrm{DNA}^{[43]}$ or proteins ${ }^{[44]}$ to fabricate 43 hydrogels capable of promoting osteogenic differentiation in 44 vitro $^{[45,46]}$ or bone regeneration in vivo in mice. ${ }^{[4]}$

Here, we report the synthesis and characterization of a 46 multicomponent self-assembling system that integrates the 47 osteogenic properties of Lap, the signaling and nanofibrous 48 structure of PAs, the proangiogenic properties of the GHK-Cu ${ }^{2+} \quad 49$ peptide, and the biocompatibility and instant gelation prop- 50 erties of Tyr-modified HA to fabricate an osteoinductive and 51 osteoconductive hydrogel for bone regeneration. The system 52 also takes advantage of both covalent (oxidative coupling) and 53 noncovalent (electrostatic) interactions to generate a mate- 54 rial that is both injectable and robust. The applicability of the 55 materials was assessed in vitro using human mesenchymal 56 stem cells (hMSCs) and human umbilical vein endothelial 57 cells (hUVECs), and in vivo using a rabbit maxillary sinus floor 58 reconstruction model.

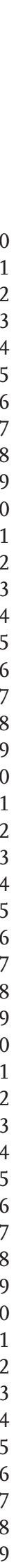




\section{Results and Discussion}

\subsection{Rationale of Design}

Our approach enables the rationale design of complex and multifunctional hydrogels for bone regeneration (Figure 1a-d). We used HA to provide a rich and biocompatible ECM macromolecule, which was functionalized with Tyr (HA-Tyr) to control stability through enzyme-mediated oxidative coupling as previously described by Eglin and co-workers. ${ }^{[47]}$ To further enhance the hydrogel's structural integrity and bioactivity, we designed PA molecules to coassemble with HA-Tyr through electrostatic interactions into nanofibers that exhibit the proangiogenic osteonectin fragment glycine-histidine-lysine $\left(\mathrm{GHK}-\mathrm{Cu}^{2+}\right) \cdot{ }^{[48]}$ In addition, Laponite discs (Lap) were incorporated to provide a distinctive dual-charged structure (i.e., positive rim and negative face charge of the nanaosilicate disc) that would facilitate interaction with both the anionic charged HA-Tyr and the cationic PA $\left(\mathrm{GHK}_{-} \mathrm{Cu}^{2+}\right)$. It is noteworthy that the osteogenic effects of Lap can be an additional benefit. Finally, to facilitate temporal control of assembly and implantation, the hydrogels were designed to assemble instantly through; a) oxidative coupling of the phenolic moiety of HA-Tyr mediated by horseradish peroxidase and $\mathrm{H}_{2} \mathrm{O}_{2}$, which has been demonstrated to be biocompatible both in vitro and in vivo, ${ }^{[49]}$ as well as b) electrostatic interactions between the cationic GHK-Cu${ }^{2+}$, anionic HA-Tyr, and anionic/cationic Lap.

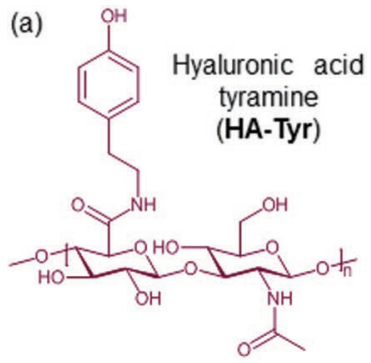

(b) Peptide amphiphile

$\left(\mathrm{GHK}-\mathrm{Cu}^{2+}\right.$

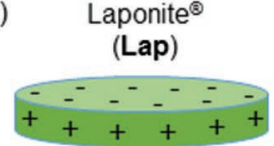

\subsection{Synthesis and Characterization of the GHK-Cu${ }^{2+}$ PA}

The PA molecule $\left(\right.$ GHK- $\mathrm{Cu}^{2+}$ ) used in this study is a three- 3 domain molecule with a hydrophobic tail (black), $\beta$-sheet 4 forming amino acid residues (blue), and the therapeutic cati- 5 onic tripeptide matrikine (pink) derived from osteonectin 6 $\left(\mathrm{C}_{16} \mathrm{H}_{31} \mathrm{CO}-\mathrm{VVVAAAGHK}\right)$ (Figure $1 \mathrm{~b}$ ). In order to present 7 GHK as a complex of copper (the form in which it exists in the 8 human body), we mixed an aqueous solution of copper (II) sul- 9 fate $\left(\mathrm{CuSO}_{4} \cdot 5 \mathrm{H}_{2} \mathrm{O}\right)\left(4 \mathrm{mg} \mathrm{mL}^{-1}\right)$ with a GHK solution (2 wt\%). 10 The copper chelating ability (GHK-Cu${ }^{2+}$ ) was confirmed by elec- 11 tron paramagnetic resonance (EPR) spectroscopy (Figure S1, 12 Supporting Information). The circular dichroism (CD) spec- 13 trum of $\mathrm{GHK}-\mathrm{Cu}^{2+}$ demonstrated a $\beta$-sheet-like bisignate 14 with a maximum and minimum at 198 and $220 \mathrm{~nm}$, respec- 15 tively (Figure S2, Supporting Information), while transmission 16 electron microscopy (TEM) confirmed self-assembly into the 17 classical PA nanofibrous morphology measuring $\approx 10 \mathrm{~nm}$ in 18 diameter and several microns in length (Figure S3, Supporting 19 Information).

\subsection{Synthesis and Characterization of the Hydrogels} prepared in stages. First, a HA-Tyr solution (6 wt\%) in horse- 26 radish peroxidase (HRP)-containing phosphate buffer saline 27
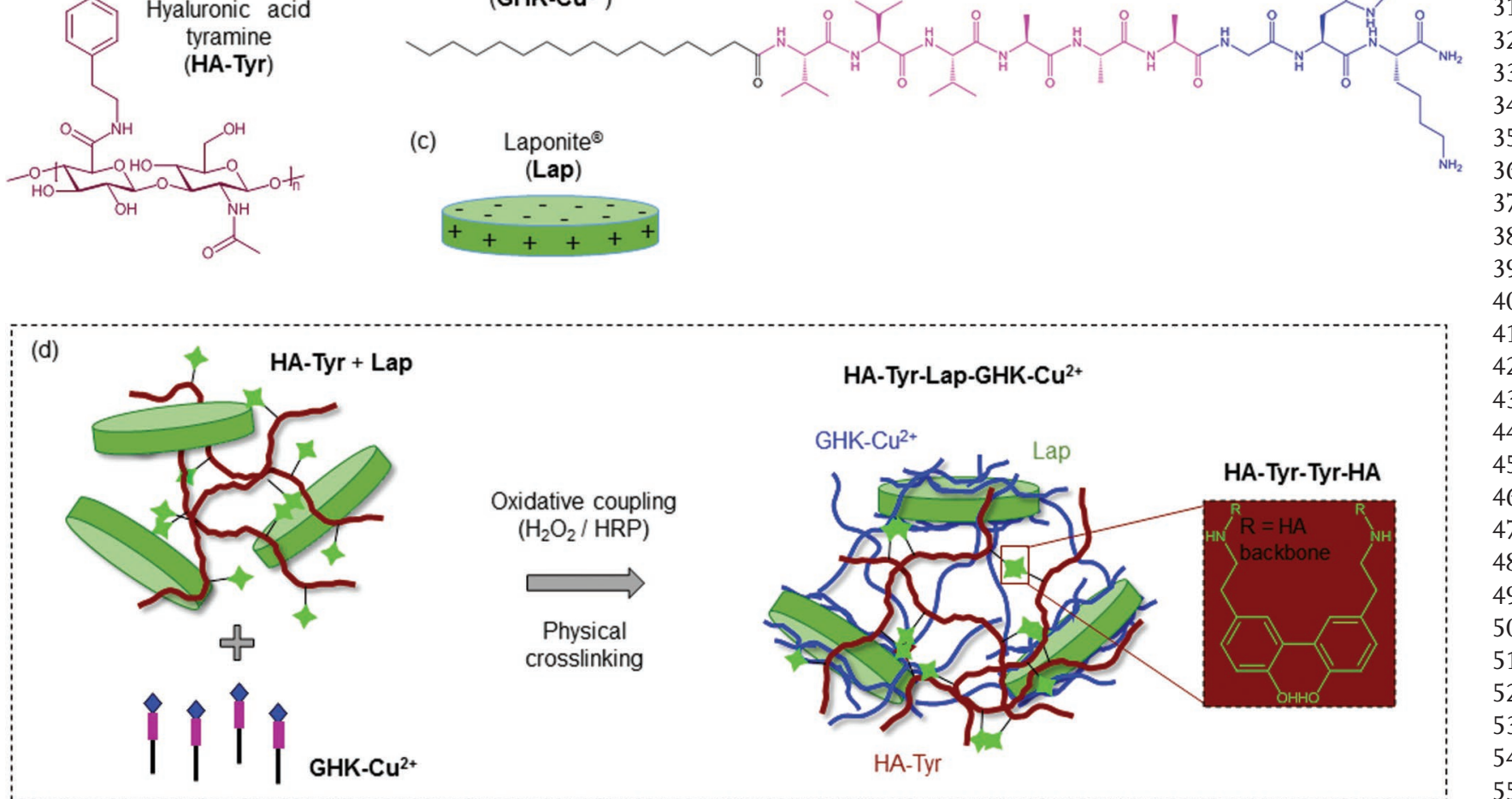

Figure 1. a) Structural formula for hyaluronic acid tyramine (HA-Tyr), b) Structural formula for peptide amphiphile (GHK), c) Structural representation 56 of Laponite (Lap) discs. d) Schematic representation of multicomponent coassembly of HA-Tyr-Lap mixtures and GHK-Cu ${ }^{2+}$ to fabricate nanocomposite 57 hydrogels via orthogonal physical crosslinking between various components and enzyme-mediated oxidative coupling of HA-Tyr to form a dityramine 58 moiety. 
(PBS) was combined with exfoliated Lap (5 wt\%). Separately, an aqueous solution of GHK-Cu${ }^{2+}(2 \mathrm{wt} \%)$ was combined with $\mathrm{H}_{2} \mathrm{O}_{2}\left(0.75 \times 10^{-6} \mathrm{M}\right)$. Upon mixing both solutions, instant gelation occurred by HRP mediated oxidative coupling of the phenolic moiety on HA-Tyr using $\mathrm{H}_{2} \mathrm{O}_{2}$ as oxidant and by electrostatic interactions between the GHK-Cu${ }^{2+}$ with both Lap and HA-Tyr. The hydrogels HA-Tyr, HA-Tyr-GHK-Cu${ }^{2+}$, and HA-TyrLap were prepared as described in the Experimental Section and used as controls. In all cases, self-supported and robust hydrogels were formed (Figure S4, Supporting Information). Due to the multicomponent nature of the hydrogels, these were prepared with fixed concentrations of both HA-Tyr ${ }^{[50]}$ and Lap $^{[45]}$ that have been previously reported by others to exhibit suitable mechanical properties and bioactivity. We used fluorescence and attenuated total reflectance-Fourier transform infrared (ATR-FTIR) spectroscopies to investigate molecular interactions underpinning coassembly of the various components in our hydrogels. The fluorescence emission spectra of HA-Tyr solution and hydrogels prepared by oxidative coupling were examined. Spectra were collected at an excitation wavelength $\left(\lambda_{\text {ex }}\right)$ of $260 \mathrm{~nm}$. The fluorescence spectra from HA-Tyr solution $(0.5 \mathrm{wt} \%)$ depicted a weak broad emission maxima $\left(\lambda_{\mathrm{em}}\right)$ at $330 \mathrm{~nm}$, which corresponds to the emission wavelength of a phenolic group (Figure S5, Supporting Information). Upon oxidative coupling the spectra shows an intense emission maxima at $422 \mathrm{~nm}$ with a shoulder at $450 \mathrm{~nm}$ (Figure S5, Supporting Information). The redshifted fluorescence emission is indicative of peroxidase-catalyzed oxidative coupling leading to dityramine/dityrosine bridge formation. ${ }^{[51]}$

The FTIR spectrum of GHK exhibited a band at $3250 \mathrm{~cm}^{-1}$ due to N-H vibrational stretching of amide II, 2952 and $2850 \mathrm{~cm}^{-1}$ due to $\mathrm{C}-\mathrm{H}$ stretching of alkyl groups, $1632 \mathrm{~cm}^{-1}$ due to $\mathrm{C}=\mathrm{O}$ stretching of amide $\mathrm{I}, 1540 \mathrm{~cm}^{-1}$ due to $\mathrm{N}-\mathrm{H}$ stretching of amide II (aromatic) and $1230 \mathrm{~cm}^{-1}$ due to $\mathrm{N}-\mathrm{H}$ stretching of amide III (Figure S6, Supporting Information). For HA-Tyr xerogel, the characteristic absorption bands were observed at 3200,1638,1540, and $1020 \mathrm{~cm}^{-1}$ corresponding to the $\mathrm{O}-\mathrm{H}$ vibrational stretching, $\mathrm{C}=\mathrm{O}$ stretching of amide $\mathrm{I}$, $\mathrm{N}-\mathrm{H}$ stretching of amide II, and $\mathrm{C}-\mathrm{O}$ asymmetric vibrational stretching, respectively (Figure S6, Supporting Information). The distinctive absorption band at $993 \mathrm{~cm}^{-1}$ in the spectrum of Lap was attributed to the Si-O vibrational stretching. ${ }^{[45]}$ In the spectrum of HA-Tyr-GHK-Cu ${ }^{2+}$ xerogel, we observed a slight shift in the vibrational stretching frequency of the $\mathrm{C}=\mathrm{O}$ region to $1635 \mathrm{~cm}^{-1}$, suggesting hydrogen bond with associated electrostatic interactions between HA-Tyr and GHK-Cu${ }^{2+}$. Similarly, the spectrum of HA-Tyr-Lap xerogel shows that the $\mathrm{C}=\mathrm{O}$ and $\mathrm{Si}-\mathrm{O}$ bands shifted to 1635 and $1000 \mathrm{~cm}^{-1}$, respectively, which is indicative of hydrogen bond interactions between HA-Tyr and Lap. Interestingly, all the changes observed in the chemical environments of the functional groups (see HA-TyrGHK and HA-Tyr-Lap) were also revealed in the spectrum of the multicomponent HA-Tyr-Lap-GHK-Cu${ }^{2+}$ xerogels. Given the evidence of the peroxidase-mediated oxidative coupling of HA-Tyr and the electrostatic and hydrogen bond interactions provided by Lap and the cationic GHK-Cu${ }^{2+}$, we reasoned that the synthesis of our multicomponent HA-Tyr-Lap-GHK-Cu ${ }^{2+}$ hydrogels is based on orthogonal interactions between all the components.

\subsection{Structural Properties of the Hydrogels}

Scanning electron microscopy (SEM) observations revealed 3 that the GHK- $\mathrm{Cu}^{2+}$ hydrogel exhibited the classical PA nano- 4 fibrous architecture ${ }^{[34]}$ (Figure 2a), while the HA-Tyr hydrogel 5 exhibited a microporous morphology (Figure 2b) as previously 6 described. ${ }^{[52]}$ As expected, HA-Tyr-GHK-Cu ${ }^{2+}$ hydrogels exhib- 7 ited features of both of these hydrogels including nanofibers 8 and microscopic pores (Figure 2c). The multicomponent HA-Tyr-Lap-GHK-Cu ${ }^{2+}$ hydrogels revealed an architecture that combined and retained all these features (Figure 2e) with Lap uniformly distributed on the surface of the fibers (Figure 2f), as evidenced by energy dispersive X-ray (EDX) analysis confirming the presence of $\mathrm{Si}, \mathrm{Mg}, \mathrm{Na}$, and $\mathrm{O}$ (Figure 2g). Although SEM examinations are not the ideal approach to describe in detail the structure of each hydrogel given the likelihood for structure disruption as a result of the sample preparation, the results provide a useful relative comparison between the different hydrogels.

\subsection{Mechanical and Physical Properties of the Hydrogels}

A major goal of our design was to provide a multifunctiona bioactive hydrogel that could stimulate specific biological processes while being a robust and easy to use system. To test the structural and mechanical properties of the hydrogels, we performed a number of tests including compressive tests, stress relaxation measurements, swelling tests, and enzymatic degradation experiments.

Compression tests were performed on $5 \mathrm{~mm}$ diameter and $5 \mathrm{~mm}$ high cylindrical hydrogels. HA-Tyr hydrogels exhibited a Young's modulus of $25.03 \pm 4.00$ (Figure 2h; and Figure S7, Supporting Information), in agreement with those of previous studies. ${ }^{[50]}$ Interestingly, upon co-assembly with GHK-Cu${ }^{2+}$ the Young's modulus of the hydrogel increased slightly up to $28.18 \pm 4.32 \mathrm{kPa}\left(\right.$ HA-Tyr-GHK-Cu${ }^{2+}$ ), which may result from an enhanced entanglement with the PA nanofibers and consequent slight decrease in porosity (Figure 2c). Importantly, upon coassembly with Lap, the hydrogels exhibited a significantly increase in Young's moduli up to $58.23 \pm 7.8 \mathrm{kPa}$ (HA-TyrLap) and $63.11 \pm 8.0 \mathrm{kPa}$ (HA-Tyr-Lap-GHK-Cu${ }^{2+}$ ) (Figure 2h, Figure S7, Supporting Information), indicating that the dityramine moieties (HA-Tyr-Tyr-HA bonds) that result from enzymatic crosslinking of the tyramine form strong physical interfacial bonds with Lap. These results are in agreement with previous studies that have reported a Lap-induced increase in the stiffness of hydrogels ${ }^{[53]}$ and evidence the potential of our hydrogels to be used as robust and bioactive hydrogel implants for bone regeneration.

It is well-established that hydrogel stiffness plays a crucial role in directing cell phenotype and that stiffer hydrogels (>30 kPa) are able to promote osteoblastic phenotypes. ${ }^{[54]}$ While the Young's modulus of our multicomponent HA-Tyr-LapGHK-Cu ${ }^{2+}$ hydrogel is higher $(63.11 \mathrm{kPa})$, differences in stiffness measuring techniques and other hydrogel properties such as porosity and stress relaxation are important to consider. In addition to the effect of hydrogel stiffness on cell behavior, stress relaxation has also been shown to play a role in cell signaling . 



Figure 2. SEM micrographs of dried xerogels of a) GHK-Cu${ }^{2+}$, b) HA-Tyr, c) HA-GHK- $\mathrm{Cu}^{2+}$, d) HA-Tyr-Lap, e) HA-Tyr-Lap-GHK-Cu ${ }^{2+}$, and f) close 4 image of HA-Tyr- Lap-GHK-Cu ${ }^{2+}$ revealing Lap nanoparticles. g) EDX elemental analysis of dried xerogels of HA-Tyr-Lap-GHK-Cu ${ }^{2+}$. h) Young's 51

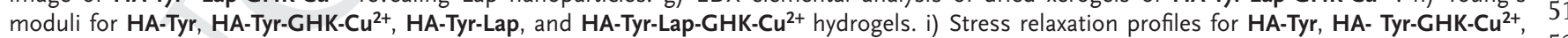
HA-Tyr-Lap, and HA-Tyr-Lap-GHK-Cu${ }^{2+}$ hydrogels. j) Swelling ratio of HA-Tyr, HA-Tyr-GHK-Cu ${ }^{2+}$, HA-Tyr-Lap, and HA-Tyr-Lap-GHK-Cu${ }^{2+}$ hydrogels. 52 k) Enzymatic degradation profiles for HA-Tyr, HA-Tyr-GHK-Cu ${ }^{2+}$, HA-Tyr-Lap, and HA-Tyr-Lap-GHK-Cu${ }^{2+}$ hydrogels. A fixed concentration (2 wt\%) of 53 GHK-Cu${ }^{2+}$ was used in all cases.

by modulating ligand binding and cytoskeletal organization. ${ }^{[55]}$ Stress relaxation tests revealed that all multicomponent hydrogels and HA-Tyr exhibited a similar relaxation profile of $\approx 55 \%$ at about $5 \mathrm{~min}$ (Figure 2i). This behavior is likely due to reversible transient molecular interactions and reorganization of the HA 56 chains and/or PA nanofibers as well as release of entangle- 57 ments driven by in situ formation of dityrosine bridges between 58 adjacent tyramine moieties in the HA-Tyr chains. However, in 59 

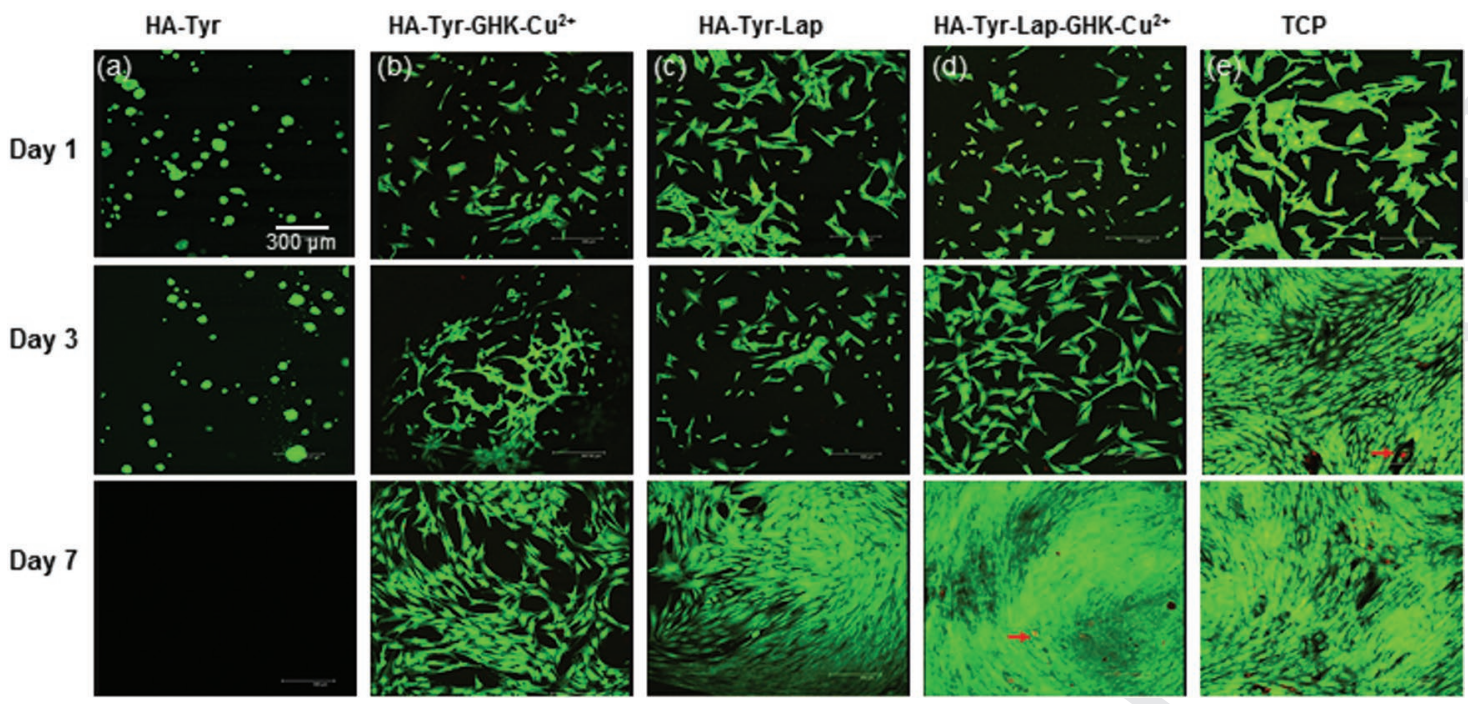

igure 3. Live/dead assay images of hMSCs seeded on a) HA-Tyr, b) HA-Tyr-GHK-Cu${ }^{2+}$, c) HA-Tyr-Lap, d) HA-Tyr-Lap-GHK-Cu${ }^{2+}$ hydrogels, and e) TCP.

the initial the $10 \mathrm{~s}$, HA-Tyr-Lap-GHK-Cu${ }^{2+}$ hydrogels exhibit a faster relaxation profile compared to all other hydrogels (Figure 2i). This rapid hydrogel stress relaxation has been shown to promote proliferation and differentiation toward an osteoblastic phenotype of mesenchymal stem cells (MSCs). ${ }^{[55]}$ These results demonstrate that the multicomponent covalent and noncovalent approach also generates a hydrogel relaxation profile with potential benefit for bone regeneration applications.

The propensity of hydrogels to absorb and retain water provides a measure of structural integrity and adaptability as well as capacity to enable critical nutrient and waste diffusion. Consequently, we conducted swelling experiments on freeze-dried hydrogels by immersing them in PBS and systematically calculating the percentage of water uptake at $0,10,20,30$, and 40 min. HA-Tyr hydrogels exhibited an exceptionally high swelling ratio of $1680 \pm 94 \%$ after 10 min of immersion in PBS, which increased to $1840 \pm 87 \%$ after 40 min (Figure 2j). Similarly, HA-Tyr-GHK-Cu ${ }^{2+}$ hydrogels displayed a high swelling ratio of $1540 \pm 57 \%$ which increased to $1802 \pm 51 \%$, after $40 \mathrm{~min}$. In contrast, Lap-containing hydrogels exhibited lower swelling ratios of $1470 \pm 45 \%$ (HA-Tyr-Lap) and $1350 \pm 17 \%$ (HA-Tyr-Lap-GHK-Cu${ }^{2+}$ ) after $10 \mathrm{~min}$ of incubation, which increased to $1540 \pm 35 \%$ (HA-Tyr-Lap) and $1580 \pm 24 \%$ (HA-Tyr-Lap-GHK-Cu${ }^{2+}$ ) after 40 min (Figure $2 \mathrm{j}$ ). This decrease suggests that despite the ionic and hydrophilic nature of Lap, its presence impedes water uptake. Since the degree of swelling ratio is inversely proportional to the crosslinking density of hydrogels, ${ }^{[56]}$ we reasoned that both the physical crosslinking provided by the Lap discs and covalent crosslinking between the tyramine moieties (HA-Tyr-Tyr-HA bonds) significantly limit water uptake in HA-Tyr-Lap and HA-Tyr-Lap-GHK-Cu ${ }^{2+}$ hydrogels. Nonetheless, all hydrogels exhibited a relatively fast and high level of equilibrium swelling ratio, returning to their original size upon rehydration (Figure $2 \mathrm{j}$ ). This capacity may facilitate their use as geometrically customized hydrogels that can be dried, stored, and rehydrated prior to implantation.

Controlled degradation is another important parameter for bioactive implantable hydrogels aiming to balance stimulation of cell growth and adequate replacement of new tissue. While HA 22 has been extensively pursued as a biomaterial, its use to fabricate 23 robust scaffolds for tissue regeneration has been limited in large 24 part by its susceptibility to rapid degradation in the presence of hya- 25 luronidases both in vitro and in vivo. ${ }^{[57]}$ Several approaches have 26 been devised to enhance HA hydrogel stability to enzymatic deg- 27 radation with varying degree of success. ${ }^{[58,59]}$ Our design enables 28 the possibility to combine covalent and noncovalent interactions 29 as well as exploit synergistic interactions between the different 30 components. To test this, hydrogels were prepared, immersed in 31 aqueous solutions of hyaluronidase $\left(20 \mathrm{U} \mathrm{mL}^{-1}\right)$ at $37^{\circ} \mathrm{C}$, and their 32 weight measured at various times points up to $50 \mathrm{~d}$ (Figure $2 \mathrm{k}$ ). 33 At $18 \mathrm{~d}$, HA-Tyr hydrogels were found to be completely digested, 34 while the multicomponent (HA-Tyr-GHK-Cu ${ }^{2+}$, HA-Tyr-Lap, 35 and HA-Tyr-Lap-GHK-Cu${ }^{2+}$ ) hydrogels resisted full degrada- 36 tion up to $50 \mathrm{~d}$ (Figure 2k). In particular, Lap-containing hydro- 37 gels exhibited a significant decrease in degradation rate, which 38 correlates with previous studies also reporting an enhanced Lap- 39 induced stability in polymers ${ }^{[60]}$ and biopolymers. ${ }^{[45]}$ We speculate 40 that the suppressed hydrogel susceptibility to rapid enzymatic 41 degradation observed in HA-Tyr-Lap and HA-Tyr-Lap-GHK-Cu ${ }^{2+} 42$ may be attributed to the strong affinity of Lap discs to proteins 43 and enzymes through physical adsorption, ${ }^{[41]}$ which would conse- 44 quently limit hyaluronidase access to the HA backbone.

\subsection{In Vitro Assessment of the Hydrogels}

\subsubsection{Viability and Proliferation of hMSCs}

To test the applicability of the multicomponent hydrogels, 52 we first conducted in vitro tests by culturing hMSCs on the 53 materials. Using a live/dead assay, cytocompatibility was 54 first assessed. As expected, HA-Tyr alone did not support cell 55 attachment and spreading (Figure 3), likely as a result of lack 56 of cell-binding motifs. ${ }^{[52]}$ However, cells cultured on all multi- 57 component HA-Tyr-GHK-Cu ${ }^{2+}$, HA-Tyr-Lap, and HA-Tyr-Lap- 58 GHK-Cu ${ }^{2+}$ hydrogels adhered and appeared to exhibit a spread 59 

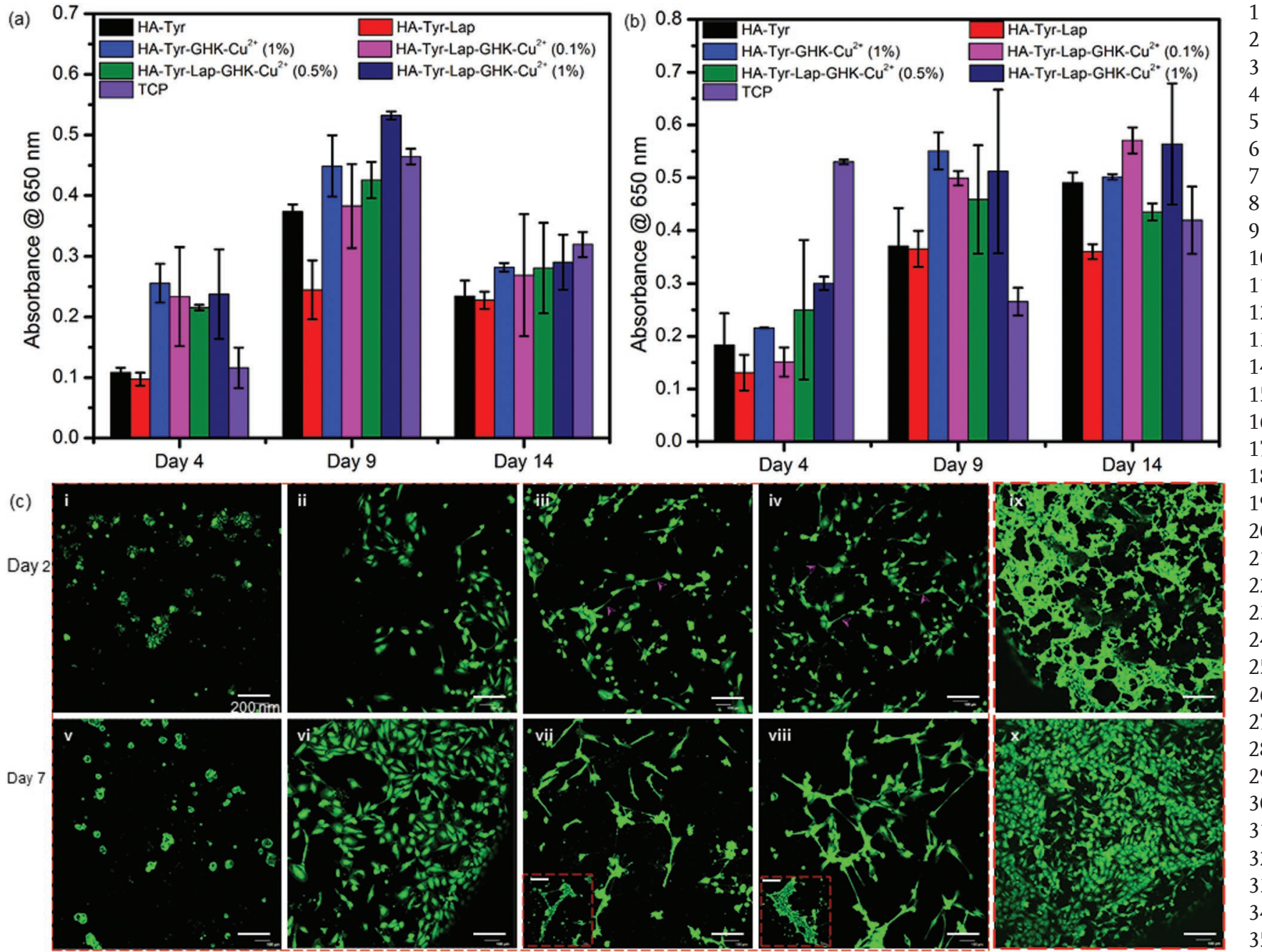

Figure 4. a) Quantitative measurement of cell proliferation on HA-Tyr, HA-Tyr-GHK-Cu+2+, HA-Tyr-Lap, HA-Tyr-Lap-GHK-Cu${ }^{2+}$ hydrogels, and TCP b) Osteogenic differentiation of hMSCs without osteoinductive media on HA-Tyr, HA-Tyr-GHK-Cu², HA-Tyr-Lap, HA-Tyr-Lap-GHK-Cü hydrogels, and TCP $(*, * *, * * *, * * * *$ indicate significant difference with respect to the color code, $p=0.01-0.001, p<0.001, p=0.001-0.0001$, and $p<0.0001$, respectively). c) CLSM images of calcein-stained hUVECs at day 1 on (i) HA-Tyr, (ii) HA-Tyr-Lap, (iii) HA-Tyr-GHK-Cu², and (iv) HA-Tyr-Lap-GHK-Cu2+ hydrogels and day 5 on (v) HA-Tyr, (vi) HA-Tyr-Lap, (vii) HA-Tyr-GHK-Cu+ (inset: large lumen), (viii) HA-Tyr-Lap-GHK-Cu2+ hydrogels, (ix) Matrigel$\operatorname{VEGF}\left(50 \mathrm{ng} \mathrm{mL}^{-1}\right)$, and (x) TCP. Insets of (vii) and (viii) are other lumen structures found on the hydrogels.

morphology (Figure 3b-d) on days 1, 3, and 7 similar to cells cultured on tissue culture plastic (TCP) (Figure 3e). We then examined the effect of the hydrogels on hMSC proliferation and found that cell number increased from day 1 to 7 on all multicomponent hydrogels, with greater increase on HA-Tyr-LapGHK-Cu ${ }^{2+}$ compared to HA-Tyr-Lap and HA-Tyr-GHK-Cu ${ }^{2+}$. In addition, cell proliferation was higher on HA-Tyr-Lap-GHK$\mathrm{Cu}^{2+}$ prepared with the higher concentration of $\mathrm{GHK}-\mathrm{Cu}^{2+}$ (1 wt\%) compared to the lower one (0.1 wt\%) (Figure 4a), which is in agreement with previous work reporting the stimulating role of GHK on cell proliferation. ${ }^{[61]}$

\subsubsection{Osteoblastic Differentiation of hMSCs}

Next, we assessed the osteoinductive potential of the multicomponent hydrogels by growing hMSCs using culture media with and without osteoinductive agents and quantifying alka- 43 line phosphatase (ALP) expression. In this case, we tested 44 three different hydrogels comprising different concentrations 45

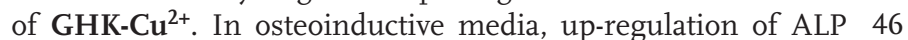
activity was observed on all hydrogels with highest expression 47 at day 4 on the TCP control compared to day 9 for the multi- 48 component hydrogels, suggesting a delayed expression on the 49 hydrogels (Figure S9, Supporting Information). However, in 50 the absence of osteoinductive media, a more indicative scenario 51 of the inductive properties of the materials, ALP expression 52 peaked for all tested substrates on day 9. In this case, the 53 highest expression was observed on the HA-Tyr-Lap-GHK-Cu ${ }^{2+} 54$ hydrogels containing the highest concentration of GHK-Cu${ }^{2+} 55$ compared to all other hydrogels and TCP control (Figure 4b). 56 This result suggests that the multicomponent HA-Tyr-Lap- 57 GHK- $\mathrm{Cu}^{2+}$ hydrogel has the capacity to stimulate osteoblastic 58 differentiation on hMSCs in the absence of osteoinductive 59 
factors. Furthermore, the dose-dependent effect of GHK-Cu${ }^{2+}$ on ALP activity is in agreement with previous reports on the osteogenic effect of copper-free alginate-GHK hydrogels. ${ }^{[61]}$

\subsubsection{Angiogenic Effect on hUVECs}

Blood vessel formation is essential in bone regeneration. Therefore, we qualitatively assessed the in vitro proangiogenic effect of the multicomponent hydrogels on hUVECs. By comparing the morphology of calcein-stained hUVECs growing on the different materials, GHK-Cu${ }^{2+}$-containing multicomponent hydrogels HA-Tyr-GHK-Cu${ }^{2+}$ and HA-TyrLap-GHK-Cu ${ }^{2+}$ were observed to trigger cell elongation and angiogenic sprouting with microcapillary-like structures by day 1 of culture (Figure 4c_(iii-iv)). In contrast, cells seeded on HA-Tyr and HA-Tyr-Lap (without GHK-Cu${ }^{2+}$ ) maintained their normal endothelial phenotype (Figure 4c_(i-ii). In addition, a more complex vascular lumen ${ }^{[62]}$ structure was also formed on the GHK- $\mathrm{Cu}^{2+}$-containing hydrogels after day 5 (Figure 4c_(vii-viii)). Such lumen structures were similar to those observed on the positive control (Matrigel $+50 \mathrm{ng} \mathrm{mL}^{-1}$ VEGF) (Figure 4c_(ix)) at day 5 . These results suggest that the GHK-Cu${ }^{2+}$-containing multicomponent hydrogels have proangiogenic properties.

The proangiogenic effects and ability of GHK to increase VEGF secretion have been previously associated with its binding to $\alpha_{6}$ or $\beta_{1}$ integrin or both. ${ }^{[63]}$ Also, a recent metabolomics pathway analysis of cells in alginate-GHK hydrogels revealed that the integrin linked kinase mediates the numerous biological functions of GHK tripeptide. ${ }^{[61]}$ The formation of vascular system is mainly ensured by the emergence of new microcapillary from existing vessels (sprouting). ${ }^{[64]}$

\subsection{In Vivo Assessment of the Hydrogels}

To test the bioactivity and in vivo bone regenerative capacity of the hydrogels, experiments were conducted on a standard sinus rabbit model following established protocols. ${ }^{[65]}$ The four hydrogel materials were tested with untreated animals serving as negative controls and animals treated with the commercial product Bio-Oss (Geistlich Ltd., Switzerland) as positive controls. Bio-Oss is a bone substitute material derived from deproteinized bovine bone marrow and has been widely used in regenerative dentistry with good success as a filler for maxillary sinus augmentation. ${ }^{[6]}$ Animals were implanted with $50 \mu \mathrm{L}$ of the hydrogels or positive control, sacrificed at either 6 or 12 weeks after implantation, and assessed qualitatively and quantitatively for bone formation and cytotoxicity.

All animals survived surgery and exhibited normal behavior during the implantation time and no signs of inflammation or infection were physically observed at the time of sacrifice. Cone beam computer tomography (CBCT) was used to qualitatively assess differences in the performance of the different materials. At 6 and 12 weeks, CBCT scans revealed new bone formation in the sinus of animals treated with the hydrogels and positive control (Bio-Oss) (Figure 5b). However, higher contrast likely associated with new bone formation was 1 observed in animals treated with the multicomponent hydro- 2 gels, especially those treated with the HA-Tyr-Lap-GHK-Cu${ }^{2+} 3$ hydrogels. Interestingly, this enhancement also appeared to be 4 larger than animals treated with the positive control at both $6 \quad 5$ and 12 weeks' time-points. It is noteworthy that the radiation 6 resistant area in the sinus of the positive control is partly due 7 to the residual materials of the Bio-oss, which can be difficult 8 to distinguish from the newly mineralized tissue. Nonetheless, the results suggest that the HA-Tyr-Lap-GHK-Cu ${ }^{2+}$ hydro- 10 gels are capable of promoting new bone formation within the 11 sinus.

To confirm this result, histological sections stained with 13 hematoxylin and eosin (HE) were acquired from animals at 14 6 and 12 weeks after implantation. Animals implanted with 15 the HA-Tyr-Lap-GHK-Cu ${ }^{2+}$ hydrogels qualitatively exhibited 16 greater amounts of newly formed bone compared to animals 17 treated with all other hydrogels and controls at both weeks $6 \quad 18$ and 12, evidenced by the presence of relevant cells and ossi- 19 fied tissue (Figure 5c). These histological sections were then 20 used to quantify new bone formation within the sinus region 21 by identifying and quantifying the areas within the sinus 22 region exhibiting osteocytes and ossified tissue (Figure 5a). At 23 both 6 and 12 weeks postsurgery, the results confirmed that 24 animals receiving the multicomponent HA-Tyr-Lap-GHK- 25 $\mathrm{Cu}^{2+}$ hydrogels revealed the highest percentage of mean area 26 of ossified tissue $(40.37 \pm 1.54$ and $60.12 \pm 2.80 \%$, respec- 27 tively) within the sinus of all tested groups including signifi- 28 cantly higher than animals treated with the positive control 29 $(35.97 \pm 1.54 \%$ and $37.56 \pm 1.18 \%$, respectively) (Figure 6a). 30 The histological sections of animals treated with the HA- 31 Tyr-Lap-GHK-Cu ${ }^{2+}$ hydrogels also exhibited both osteoblasts 32 and osteocytes within lacuna, further evidencing the pres- 33 ence of an active regenerative environment (Figure 6b). It is 34 likely that these cells emerged from MSCs or preosteoblasts 35 migrating from the local bone surface, periosteum, or the 36 blood. By comparing these results with those of the other 37 hydrogels (Figure 6a), we conclude that the enhanced bioac- 38 tivity of HA-Tyr-Lap-GHK-Cu ${ }^{2+}$ hydrogels may result primarily 39 from the presence of Lap and GHK-Cu${ }^{2+}$. These results cor- 40 relate with the in vitro results, which show that Lap and espe- 41 cially GHK may have osteoinductive properties in the absence 42 of growth factors. Vascularization is of upmost importance 43 in bone regeneration. Closer examination of the histological 44 sections revealed that animals treated with HA-Tyr-Lap-GHK- 45 $\mathrm{Cu}^{2+}$ exhibited a qualitatively higher amount of blood vessels 46 compared to animals treated with HA-Tyr-GHK-Cu${ }^{2+}$ and the 47 positive control (Figure 6c). Interestingly, blood vessels were 48 much less prevalent in hydrogels that did not contain GHK- 49 $\mathrm{Cu}^{2+}$, which is in accordance with the in vitro experiments as 50 well as previous studies that have reported on the ability of 51 GHK-Cu ${ }^{2+}$ to promote formation of blood vessels in mice. ${ }^{[66]} 52$ The in vivo experiments were also used to investigate the 53 biosafety of the hydrogels through a systemic toxicity test. His- 54 tological sections of the liver, heart, spleen, lung, and kidney 55 from the animals treated with the HA-Tyr, HA-Tyr-GHK-Cu${ }^{2+}, \quad 56$ HA-Tyr-Lap, and HA-Tyr-Lap- GHK-Cu ${ }^{2+}$ hydrogels were exam- 57 ined and presented no signs of inflammation or histological 58 changes compared to control animals (Figure S11, Supporting 59 

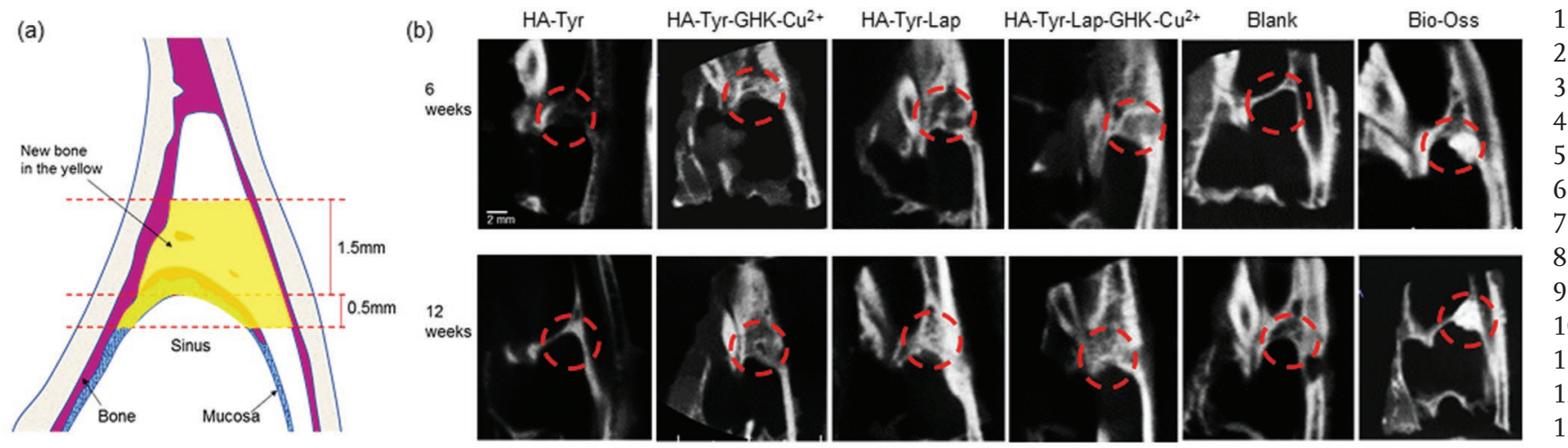

(c) HA-Tyr
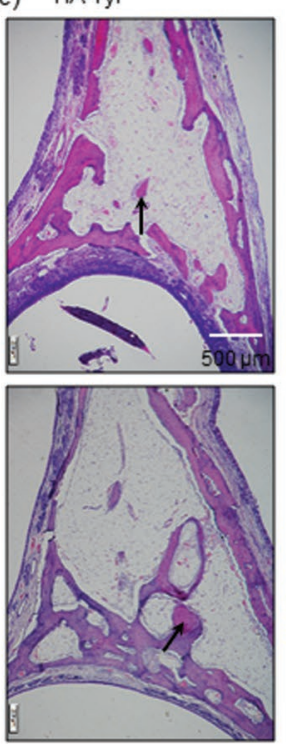

HA-Tyr-GHK-Cu2+
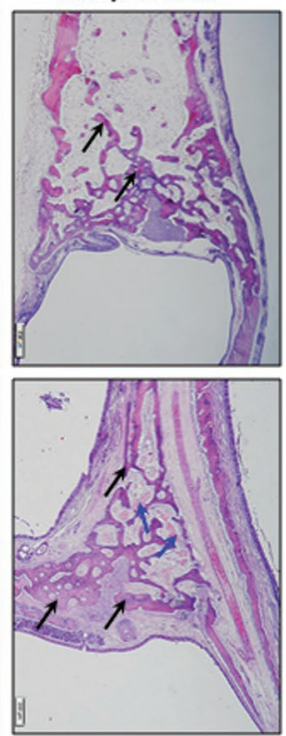

HA-Tyr-Lap


HA-Tyr-Lap-GHK-Cu2+
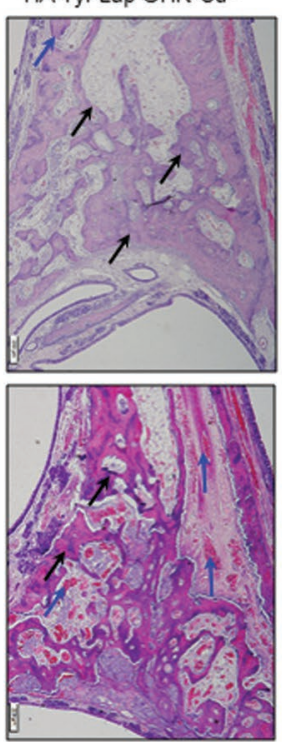

Blank
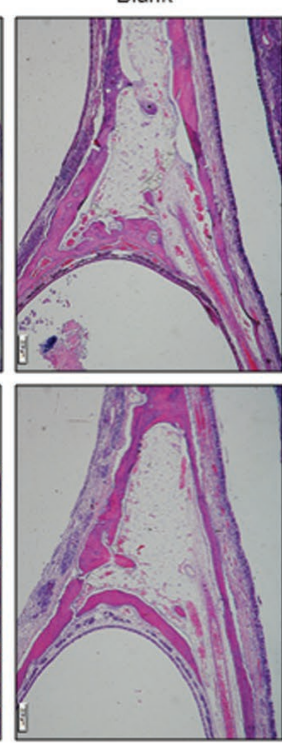

Bio-Oss


Figure 5. a) Schematic representation of new bone area in the maxillary sinus. b) Cone beam computed tomography (CBCT) scan results of sinus 35 floor treated with HA-Tyr, HA-Tyr-GHK-Cu${ }^{2+}$, HA-Tyr-Lap, and HA-Tyr-Lap-GHK-Cu${ }^{2+}$ hydrogels compared with sinus treated with nothing and those 36 treated with Bio-Oss after 6 and 12 weeks postsurgery. c) New bone formation detected by hematoxylin and eosin (HE) staining shows local histological 37 images of the different groups at 6 and 12 weeks postsurgery, respectively. The black, blue, and orange arrows indicate the new trabecular bone and 38 blood vessels filed with red blood cells and residual Bio-Oss.

Information). These results suggest that the HA-Tyr-Lap-GHK$\mathrm{Cu}^{2+}$ hydrogels did not degrade into toxic by-products. We speculate that, beyond the molecular signaling and biocompatibility of the HA-Tyr-Lap-GHK-Cu ${ }^{2+}$ hydrogel, its high osteo-promoting activity may also result from the inherent mechanical and physical properties of the hydrogel. Both the hydrogel's Young's modulus (Figure $2 \mathrm{~h}$ ) and stress relaxation profile (Figure 2i) exhibit values that have been reported to be beneficial for bone promoting applications. ${ }^{[5,55]}$ Furthermore, the hydrogels display an enzymatic degradation profile (Figure $2 \mathrm{k}$ ) that may have permitted sufficient signaling to migrating and surrounding cells while progressively degrading to enable new tissue formation. This capacity for timely scaffold degradation is key for optimum tissue regeneration. ${ }^{[67]}$ Furthermore, beyond these beneficial molecular, chemical, and physical properties, the potential to easily manipulate and deliver the hydrogel represent key advantages to facilitate its clinical use and impact.

\section{Conclusion}

We have developed a practical and multifunctional self-assembling hydrogel biomaterial for bone regeneration applications. 45 The material takes advantage of both covalent and noncova- 46 lent interactions to integrate HA, PAs, and Lap into a bioactive 47 hydrogel with a spectrum of molecular, physical, and mechan- 48 ical properties designed to promote bone regeneration as 49 well as minimally invasive implantation. We demonstrate the 50 capacity of the hydrogels to support cell growth and stimulate 51 both osteoblastic differentiation and angiogenic sprouting of 52 hUVECs in vitro as well as promote faster bone regeneration 53 in a rabbit model compared to a commercially available gold- 54 standard material. The current study introduces a new molecu- 55 larly designed self-assembling material that stimulates bone 56 formation without the use of exogenous growth factors and 57 demonstrated its potential use in maxillary sinus reconstruc- 58 tion and other bone tissue regeneration procedures. 


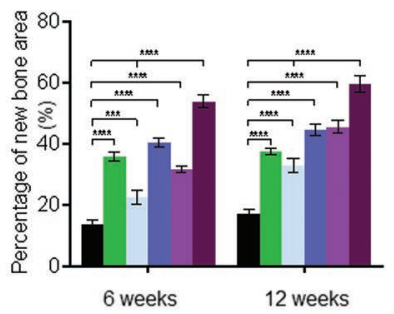

$$
\begin{aligned}
& \text { Blank } \\
& \text { Bio-oss } \\
& \text { HA-Tyr } \\
& \text { HA-Tyr + GHK-Cu2+ } \\
& \text { HA-Tyr + Lap } \\
& \text { HA-Tyr + Lap + GHK-Cu2+ }
\end{aligned}
$$


Figure 6. a) Calculated new bone area in the sinus treated with HA-Tyr, HA-Tyr-GHK-Cu${ }^{2+}$, HA-Tyr-Lap and HA-Tyr-Lap-GHK-Cu${ }^{2+}$ hydrogels compared with the group treated with nothing (negative control) and those treated with Bio-Oss (positive control) at 6 and 12 weeks (*indicates significant differences, $p<0.001)$. Hematoxylin and eosin staining shows local histological image of the group treated with the multicomponent HA-Tyr-Lap-GHK-Cu ${ }^{2+}$ hydrogels at 12 weeks postoperation. b) Histological image showing osteocytes in the trabecular bone (red arrows) and osteoblasts on the surface of the new trabecular bones (yellow arrows) in the group treated with HA-Tyr-Lap-GHK-Cu${ }^{2+}$ hydrogels. c) The blue arrows and yellow circles indicate the blood vessels filed with red blood cells within the new bones and foam cell-like which engulfed the implanted HA-Tyr-Lap-GHK-Cu ${ }^{2+}$ hydrogels.

\section{Experimental Section}

Materials: Laponite XG was a generous gift from the laboratory of Professor Richard OC Oreffo and Dr. Jonathan Dawson, University of Southampton while HA-Tyr was synthesized as previously described elsewhere. ${ }^{[45]}$ Dulbecco's Modified Eagle's Medium (DMEM), fetal bovine serum (FBS), PBS, penicillin, and streptomycin, horseradish peroxidase, hydrogen peroxide were purchased from sigma-Aldrich Inc. (UK). The Live/Dead assay kits (calcein AM and ethidium homodimer) were purchased from Thermo Fisher Scientific Ltd (UK).

Peptide Synthesis and Characterization: PAs were synthesized as previously described ${ }^{[68]}$ by solid phase peptide synthesis on Liberty Blue-automated microwave peptide synthesizer (CEM Ltd, UK). The standard 9-fluorenylmethoxycarbonyl (Fmoc) protection chemistry on a 4-methylbenzhydrylamine (MBHA) Rink Amide resin (Novabiochem Corporation, UK) was employed. Amino acid couplings were performed using $4 \mathrm{mmol}$ equivalent of Fmoc-protected amino acids (Novabiochem Corporation, UK), $4 \mathrm{mmol}$ equivalents of 1-hydroxybenzotriazol (HOBT, Carbosynth Ltd, UK) and $6 \mathrm{mmol}$ equivalents of $N, N$ '-diisopropylcarbodiimide (DIC, Sigma-Aldrich Inc., UK) for $1 \mathrm{~h}$. Fmoc deprotections were performed with 20\% piperidine (Sigma-Aldrich Inc., UK) in N,N-dimethylformamide (DMF, Alfa Aesar Inc., UK). Following Fmoc removal from the final amino acid residue, the alkyl tail moiety (from palmitic acid, $\mathrm{C}_{16} \mathrm{H}_{32} \mathrm{O}_{2}$, Calbiochem Inc., UK) was conjugated to the free $\mathrm{N}$-terminus. The alkylation reaction was accomplished by using palmitic acid $(4 \mathrm{mmol})$, HOBT $(4 \mathrm{mmol})$, and DIC $(6 \mathrm{mmol})$ in $\mathrm{DMF} /$ dichloromethane. The reaction was allowed to proceed at room temperature for $4 \mathrm{~h}$ or until obtaining a negative Kaiser test. PA cleavage from the resin and deprotection of the side chains were carried out with a mixture of trifluoracetic acid (TFA, Sigma-Aldrich Inc., UK)/triisopropylsilane (TIS, Alfa Aesar Inc., UK)/water (95:2.5:2.5) for $3 \mathrm{~h}$ at room temperature. After filtration of the cleavage mixture, TFA was removed by rota-evaporation and the resulting solution was triturated with cold diethylether at $-20^{\circ} \mathrm{C}$. The precipitate was collected by centrifugation, washed twice with cold diethylether, air-dried, dissolve in deionised water and lyophilized.
The product was then purified using a preparative HPLC (Waters Ltd, USA) with reverse-phase Xbridge C18 column (Waters Ltd, USA) and water/acetonitrile $(0.1 \%$ TFA) binary mobile phase. Hydrochloric acid $\left(10 \times 10^{-3} \mathrm{M}\right)$ was added to the HPLC fractions and rotavap to remove the residual TFA, leaving behind chloride as the PA counter ions. Finally, the PA was dialyzed against deionized water using $500 \mathrm{MWCO}$ dialysis tubing (Spectrum Europe BV, The Netherlands) to remove salts, lyophilized to obtain a white fluffy pure PA. Circular dichroism (CD) was measured with Chirascan circular dichroism spectrometer (Applied Photophysic Ltd, UK) using quartz cell with $1 \mathrm{~mm}$ path length and the following parameters: data pitch $-0.5 \mathrm{~nm}$, scanning mode - continuous, scanning speed $-100 \mathrm{~nm} \mathrm{~min}^{-1}$, bandwith $-2 \mathrm{~nm}$ and accumulation-5. All CD data were presented as ellipticity and recorded in millidegree (mdeg). CD spectra were obtained by signal integrating 3 scans, from 190 to $260 \mathrm{~nm}$ at speed of $50 \mathrm{~nm} \mathrm{~min}$. . Data were processed by a simple moving average and smoothing method. Electron paramagnetic resonance spectroscopy was recorded on a Bruker EMX EPR equipped with a standard cavity, operating at X-band frequency using standard Wilmad quartz tubes at $-80^{\circ} \mathrm{C}$. Transmission electron microscopy was performed on etched carbon-coated copper grids (Agar Scientific Ltd, Stanstead, UK) using JEOL 1230 TEM fitted with Morada CCD camera. Samples were stained with aqueous $2 \%$ uranyl acetate solution.

Hydrogel Preparation and Characterizations: The requisite concentration (6 wt\%) of HA-Tyr was prepared in PBS containing 3 unit $\mathrm{mL}^{-1}$ of HRP and allowed to fully dissolve overnight at $4{ }^{\circ} \mathrm{C}$. This concentration of HA-Tyr was used in all the hydrogel preparations. Gelation of the HA-Tyr was triggered by adding aqueous solution of $\mathrm{H}_{2} \mathrm{O}_{2}\left(0.75 \times 10^{-3} \mathrm{M}\right)$ and gentle mixing with a pipette tip. In order to prepare HA-Tyr-GHK-Cu${ }^{2+}$ hydrogels, aqueous solution of $\mathrm{CHK}_{-} \mathrm{Cu}^{2+}(2 \mathrm{wt} \%)$ with $0.4 \mathrm{mg} \mathrm{mL}^{-1}$ $\left.\mathrm{CuSO}_{4}\right)$ prepared in $\mathrm{H}_{2} \mathrm{O}_{2}(\mathrm{aq})\left(0.75 \times 10^{-3} \mathrm{M}\right)$ was added to HA-Try/ HRP solution with a quick mixing. To synthesize HA-Tyr-Lap hydrogels, aqueous suspension of Lap (5 wt\%) exfoliated with sodium salt of polyacrylic acid $\left(\approx 5000 \mathrm{Da} M_{w} 0.6\right.$ wt $\left.\%\right)$ was initially mixed with aqueous solution HA-Tyr/HRP followed by the addition of $\mathrm{H}_{2} \mathrm{O}_{2}$ (aq) $\left(0.75 \times 10^{-3} \mathrm{M}\right)$. Similarly, HA-Tyr-Lap-GHK-Cu${ }^{2+}$ hydrogels were prepared 
by adding aqueous solution of $\mathrm{GHK}-\mathrm{Cu}^{2+} / \mathrm{H}_{2} \mathrm{O}_{2}$ to HA-Tyr/HRP/Lap mixture and mixed with a pipette tip.

Molecular Characterization of Coassembly: IR spectra were recorded on a PerkinElmer ATR-FTIR spectrometer in the range of $4000-400 \mathrm{~cm}^{-1}$ at $2 \mathrm{~cm}^{-1}$ resolution. Fluorescence spectroscopy was carried out on a Hitachi F4500 spectrophotometer. Both excitation and emission slit width was $10.0 \mathrm{~nm}$, scan speed was set to $200 \mathrm{~nm} \mathrm{~min}{ }^{-1}$.

Unconfined Compression and Stress Relaxation Testing: Elastic moduli Eo and stress relaxation properties of the hydrogels were measured from the compression tests of the hydrogels with an Instron 560 (Instron, Norwood, MA) using $10 \mathrm{~N}$ load cell to a compressive strain of $30 \%$ and a deformation rate of $1 \mathrm{~mm} \mathrm{~s}^{-1}$. The cylindrical hydrogels (diameter $=$ height $=5 \mathrm{~mm}$ ) were swollen in PBS for $6 \mathrm{~h}$ prior measurements and the testing was carried out inside a flat-bottom petridish filled with PBS. Prior to the tests, an initial compressive contact of $0.01 \mathrm{~N}$ was applied to ensure a complete contact between the hydrogels and the petri-dish. No bulging of the side faces of the hydrogels was observed. The slope of the stress versus strain curve gives Eo. After the compression test, the strain was held constant for $5 \mathrm{~min}$, while the load was recorded as a function of time. In order to calculate the stress, the force was divided by the area of the hydrogels in the undeformed state. Tests were carried out three times to ensure reproducibility.

Swelling Properties and Enzymatic Digestion of Hydrogels: In order to determine the swelling properties of the hydrogels, the wet hydrogels were first freeze-dried to a constant initial weight $\left(W_{i}\right)$. The dried xerogels were then incubated in PBS at $37^{\circ} \mathrm{C}$. The wet weights $(\mathrm{W} / \mathrm{t})$ of the hydrogels were measured at various time intervals during incubation until there was no noticeable increase in the weight the wet hydrogels. The percentage swelling of the hydrogels was calculated using the following equation $\left.\left(W_{t}-W_{i}\right) / W_{i} \times 100\right)$. Degradation rate of the hydrogels was characterized by incubating the cylindrical hydrogels in PBS $(\mathrm{pH}=7.2)$ for $24 \mathrm{~h}$ and then treated with hyaluronidases $\left(20\right.$ unit $\left.\mathrm{mL}^{-1}\right)$. The remaining weight of the hydrogels was measured up to $50 \mathrm{~h}$ after enzymatic treatment. The weight loss was computed using equation $\left(W_{f}-W_{i}\right) / W_{i} \times 100$, where $W_{i}$ and $W_{f}$ represent initial and final hydrogel weights, respectively.

Characterization of Microstructures: Microstructure of the hydrogels was observed using SEM. The hydrogels were frozen by liquid nitrogen and lyophilized to obtain dried samples. The dried gels were then sputter-coated with gold (10 nm thick) for $60 \mathrm{~s}$. SEM micrographs of the dried xerogels were acquired on Inspect F50 (FEl Comp, the Netherlands). EDX spectroscopy was also used to analyze the dried gels coated with carbon to obtain elemental compositions of the dried xerogels.

In Vitro Experiments: Live/Dead assay_Live/dead assay was performed using a Live/Dead Cytotoxicity Kit (Thermo Fisher Scientific, UK) hMSCs (5000 cells $\mathrm{mL}^{-1}$ ) (Thermo Fisher Scientific, UK) seeded on various hydrogels at day 1, 3, and 7. Imaging was performed on an inverted confocal laser scanning microscope (CLSM, Leica, Germany). Proliferation-Cell proliferation was assessed by seeding $5000 \mathrm{hMSC}$ on various hydrogels. The cell seeded hydrogels were seeded incubated at $37{ }^{\circ} \mathrm{C}$ under $5 \% \mathrm{CO} 2$ condition. Cell proliferation was quantitatively measured at various time points (day 1, 4, and 7) using PrestoBlue reagent (PrestoBlue Cell Viability Reagent, Thermo Fisher Scientific, UK). Briefly, the spent media was removed from each well and the cells were incubated with PrestoBlue reagent solution $(100 \mu \mathrm{L})$. The cells were incubated for $1 \mathrm{~h}$ at $37^{\circ} \mathrm{C}$, away from light. Florescence measurements were carried out with using excitation wavelength of $560 \mathrm{~nm}$ and an emission wavelength of $590 \mathrm{~nm}$. Differentiation-Cell differentiation was assessed by seeding 20000 hMSCs (passage 10) on various hydrogels with and without osteogenic media. Experiments were setup in 4 triplicates. With osteogenic media, cells were first cultured with basal media and replaced with osteogenic media after $1 \mathrm{~d}$. After each time point, cells were fixed using $4 \%$ formaldehyde for $10 \mathrm{~min}$ and washed thrice with sterile $\mathrm{dH} 2 \mathrm{O}$. Cells were incubated with $100 \mu \mathrm{L}$ of SIGMAFAST BCIP/NBT reagent (Sigma, UK). ALP activity was spectroscopically quantified after days 4,9 , and 14 by measuring absorbance at $650 \mathrm{~nm}$. Angiogenesis-hUVECs (5000) were seeded on various hydrogels. Cell-seeded hydrogels were incubated with 1 supplemented endothelial cells growth media for 1 and $5 \mathrm{~d}$. Cells were 2 stained with calcein $\mathrm{AM}\left(2 \times 10^{-3} \mathrm{M}\right)$ and optical images were acquired on CLSM (Leica, Germany).

In Vivo Maxillary Sinus Floor Reconstruction Procedure: 30 healthy 4 adult New Zealand rabbits were randomly divided into two study 5 groups (control and experimental groups) for observation at week 6 and 6 week 12. General anesthesia was performed through injection of 3\% 7 sodium pentobarbital via the marginal ear vein $\left(1 \mathrm{~mL} \mathrm{~kg}^{-1}\right)$. After the 8 disappearance of eyelash reflex, the hair in the surgery region was shaved 9 and the region was sterilized by $1 \%$ lodine volts. A $2.5 \mathrm{~cm}$ incision on the nasal skin along the midline was made and the periosteum was stripped to expose the nasal bone and nasoincisal suture line. Two round windows at each side of the midline were prepared using bone drill. The windows were $5 \mathrm{~mm}$ in diameter and located $\approx 20 \mathrm{~mm}$ anterior to the nasofrontal suture line and $10 \mathrm{~mm}$ lateral to the midline. During the osteotomy, sterile saline solution was injected to the drill for continuous cooling and the sinus membrane was carefully protected. The mucosa and periosteum were gently elevated with a periosteum elevator and $50 \mu \mathrm{L}$ gel was prepared in situ within the space upon the surface of the bone. In the experimental groups, four types of gel, as subgroups, including HA-Tyr, HA-Tyr-GHK-Cu${ }^{2+}$, HA-Tyr-Lap, and HA-Tyr- Lap-CHK-Cu ${ }^{2+}$ was formed within the space. The space in the 20 positive control group was filled with equal volume of Bio-oss and the 21 space in blank control group was empty. The periosteum and skin were then sutured. Each group consisted of five animals.

Histological and Histomorphomeric Analysis: The animals were 23 euthanized 6 and 12 weeks postsurgery by injecting sodium pentobarbital 24 via the marginal ear vein $\left(100 \mathrm{mg} \mathrm{kg}^{-1}\right)$, and the sinuses were retrieved 25 in blocks. The specimens were immediately fixed with paraformaldehyde 26 (4\%) for $48 \mathrm{~h}$ and demineralized by soaking them in 15\% disodium 27 ethylene diamine tetraacetate for 2 months and then washed in tap 28 water for $15 \mathrm{~min}$. All the specimens were trimmed and put into $70 \%$, 28 $80 \%, 90 \%, 95 \%, 100 \%$ ethanol step by step for gradually dehydrate, and finally embedded in paraffin. Longitudinal histological HE slices of $4 \mu \mathrm{m}$ thickness were obtained to visualize the entire sinus. The sections were observed and digitally captured with a microscopic imaging system composed of a microscope (BX51, Olympus, Japan) and an image processing software, Cell Sens (Olympus, Japan). For the calculation of new bone area, Photoshop (Adobe Inc.) was first used to draw the outline of the new bone and Image) Fiji (developed by the National Institutes of Health and the Laboratory for Optical and Computational Instrumentation) was then used to calculate the new bone areas. Five slices were analyzed for each group. Fresh tissue blocks containing main organs including heart, liver, spleen, lung, and kidney were obtained and fixed with $4 \%$ paraformaldehyde to prepare HE stained slices analysis.

Statistical Analysis: Statistical comparisons between groups treated with various hydrogels, blank (negative control), and Bio-oss (positive control group) were performed using 2-way ANOVA followed by Bonferroni tests if significant differences were observed using Graph Prism software (San Diego). Tukey's multiple comparison test was used for the cell proliferation and differentiation.

\section{Supporting Information}

Supporting Information is available from the Wiley Online Library or 50 from the author.

\section{Acknowledgements}

B.O.O. and S.N. contributed equally to this work. The work was 55 supported by the ERC Starting Grant (STROFUNSCAFF) and the UK 56 Regenerative Medicine Platform (UKRMP2) Acellular Smart Materials. 57 R.O. and J.D. gratefully acknowledge funding support from the UK 58 Regenerative Medicine Platform Hub Acellular SMART materials 3D 59 
architecture (MR/R015651/1) and the UK Regenerative Medicine Platform (MR/L012626/1 Southampton Imaging). J.D. thankfully acknowledge EPSRC for a fellowship (EP/L010259/1). H.S. acknowledge funding support from the National Key Research and Development Program of China (No. 2016YFC1102800) and the National Natural Science Foundation of China (No. 81870741). The authors thank Dr. Stephen Thorpe at the School of Engineering and Materials Science, QMUL for help with interpreting the mechanics of the hydrogels. They also thank Dr. Vicente Araullo-Peters and Dr. Roberto Buccafusca at Nanovision and the School of Biological and Chemical Sciences (SBCS), QMUL for technical support.

\section{Conflict of Interest}

The authors declare no conflict of interest.

\section{Keywords}

bone formation, cranio-maxillofacial surgery, multicomponent selfassembly, nanocomposite hydrogels, nanosilicates, self- assembling peptides

Received: July 30, 2019 Revised: January 3, 2020 Published online:

[1] M. Morawska-Kochman, K. Marycz, K. Jermakow, K. Nelke, W. Pawlak, M. Bochnia, PLoS One 2017, 12, e0176776.

[2] T. Starch-Jensen, J. D. Jensen, J. Oral Maxillofac. Surg. 2017, 8, e3.

[3] M. Yan, R. Liu, S. Bai, M. Wang, H. Xia, J. Chen, Sci. Rep. 2018, 8, 1451.

[4] T. M. Jeong, J. K. Lee, Maxillofac. Plast. Reconstr. Surg. 2014, 36, 146.

[5] S. Nasr, D. E. Slot, S. Bahaa, C. E. Dörfer, K. M. Fawzy El-Sayed, J. Craniomaxillofac. Surg. 2016, 44, 1607.

[6] T. Starch-Jensen, A. Mordenfeld, J. P. Becktor, S. S. Jensen, Implant Dent. 2018, 27, 363.

[7] X. Liu, P. Wang, W. Chen, M. D. Weir, C. Bao, H. K. Xu, Acta Biomater. 2014, 10, 4484.

[8] A. Dasmah, M. Hallman, L. Sennerby, L. Rasmusson, Clin. Implant Dent. Relat. Res. 2012, 14, 259.

9] A. C. Profeta, C. Huppa, Craniomaxillofac. Trauma Reconstr. 2016, 9,1

[10] A. C. Jayasuriya, A. Bhat, J. Tissue Eng. Regener. Med. 2010, 4, 340.

[11] A. Scarano, F. Lorusso, G. Staiti, B. Sinjari, A. Tampieri, C. Mortellaro, Front. Physiol. 2017, 8, 565.

12] B. Gaihre, S. Uswatta, C. A. Jayasuriya, J. Funct. Biomater. 2017, $8,49$.

[13] Z. Sheikh, S. Najeeb, Z. Khurshid, V. Verma, H. Rashid, M. Glogauer, Materials 2015, 8, 9.

[14] D. P. Link, J. van den Dolder, J. G. C. Wolke, J. A. Jansen, Tissue Eng. 2007, 13, 493

[15] F. Schwarz, A. Schmucker, J. Becker, Clin. Oral Implants Res. 2017, $28,779$.

16] D. Gothard, E. L. Smith, J. M. Kanczler, C. R. Black, J. A. Wells, C. A. Roberts, L. J. White, O. Qutachi, H. Peto, H. Rashidi, M. M. Stevens, A. J. El-Haj, F. R. Rose, K. M. Shakesheff, R. O. Oreffo, PLoS One 2015, 10, e0145080.

[17] A. C. Mendes, K. H. Smith, E. Tejeda-Montes, E. Engel, R. L. Reis, H. S. Azevedo, A. Mata, Adv. Funct. Mater. 2013, 23, 430.

[18] S. Elsharkawy, M. Al-Jawad, M. F. Pantano, E. Tejeda-Montes, K. Mehta, H. Jamal, S. Agarwal, K. Shuturminska, A. Rice,
N. V. Tarakina, R. M. Wilson, A. J. Bushby, M. Alonso, 1 J. C. Rodriguez-Cabello, E. Barbieri, A. del Roi Hernández, 2 M. M. Stevens, N. M. Pugno, P. Anderson, A. Mata, Nat. Commun. 2018, 9, 2145.

[19] E. Tejeda-Montes, A. Klymov, M. R. Nejadnik, M. Alonso, J. C. Rodriguez-Cabello, X. F. Walboomers, A. Mata, Biomaterials 2014, 35, 8339 .

[20] S. Shimizu, S. Tsuchiya, A. Hirakawa, K. Kato, M. Ando, M. Mizuno, M. Osugi, K. Okabe, W. Katagiri, H. Hibi, BMC Oral Health 2019, $19,69$.

[21] P. A. Parmar, L. W. Chow, J.-P. St-Pierre, C.-M. Horejs, Y. Y. Peng, 10 J. A. Werkmeister, J. A. M. Ramshaw, M. M. Stevens, Biomaterials 11 2015, 54, 213.

[22] H. Lopez Hernandez, A. K. Grosskopf, L. M. Stapleton, C. Agmon, E. A. Appel, Macromol. Biosci. 2019, 19, 1800275

[23] J. H. Lee, M. Y. Ryu, H.-R. Baek, H.-K. Lee, J.-H. Seo, K. M. Lee, A. Y. Lee, G. B. Zheng, B.-S. Chang, C.-K. Lee, Artif. Organs 2014, $38,149$.

[24] E. Martínez-Sanz, O. P. Varghese, M. Kisiel, T. Engstrand, 17 K. M. Reich, M. Bohner, K. B. Jonsson, T. Kohler, R. Müller, 18 D. A. Ossipov, J. Hilborn, J. Tissue Eng. Regener. Med. 2012, 6, s15. 19

[25] W. Zhang, X. Wang, S. Wang, J. Zhao, L. Xu, C. Zhu, D. Zeng, 20 J. Chen, Z. Zhang, D. Kaplan, X. Jiang, Biomaterials 2011, 32, 9415.21

[26] T.-M. De Witte, L. E Fratila-Apachitei, A. A. Zadpoor, N. A, Peppas, 22 Regener. Biomater. 2018, 5, 197.

[27] P. S. Lienemann, M. P. Lutolf, M. Ehrbar, Adv. Drug Delivery Rev. 2012, 64, 1078.

[28] J. D. Kretlow, S. Young, L. Klouda, M. Wong, A. G. Mikos, Adv. Mater. 2009, 21, 3368.

[29] B. O. Okesola, A. Mata, Chem. Soc. Rev. 2018, 47, 3721. D. K. Smith, D. J. Adams, A. Mata, Chem. Mater. 2019, 31, 7883.

[31] M. Liu, X. Zeng, C. Ma, H. Yi, Z. Ali, X. Mou, S. Li, Y. Deng, N. He, 30 Bone Res. 2017, 5, 17014.

[32] P. Y. Shona, M. Kurisawa, S. Gao, J. E. Chung, J. Y. Ying, Biomaterials 32 2009, 30, 822.

33] S. C. Dennis, J Whitlow M. S Detamore, S. L. Kieweg, C. J. Berkland, Langmuir 2017, 33, 206.

[34] M. P. Hendricks, K. Sato, L. C. Palmer, S. I. Stupp, Acc. Chem. Res. 2017, 50, 2440

[35] A. Mata, Y. Geng, K. J. Henrikson, C. Aparicio, S. R. Stock, R. L. Satcher, S. I. Stupp, Biomaterials 2010, 37, 6004

[36] Z. Huang T. D Sargeant J. F. Hulvat A. Mata, P. Bringas Ir. 39 C.-Y. Koh, S. I. Stupp, M. L. Snead, J. Bone Miner. Res. 2008, 23, 40 1995.

[37] R. N. Shah, N. A. Shah, M. M. Del Rosario Lim, C. Hsieh, G. Nuber, S. I. Stupp, Proc. Natl. Acad. Sci. USA 2010, 107, 3293.

[38] L. W. Chow, R. Bitton, M. J. Webber, D. Carvajal, K. R. Shull, A. K. Sharma, S. I. Stupp, Biomaterials 2011, 32, 1574.

[39] S. Unterman, L. F. Charles, S. E. Strecker, D. Kramarenko, D. Pivovarchik, E. R. Edelman, N. Artzi, ACS Nano 2017, 11, 2598.

[40] J. I. Dawson, R. O. C. Oreffo, Adv. Mater. 2013, 25, 4069.

[41] M. Mousa, N. D. Evans, R. O. C. Oreffo, J. I. Dawson, Biomaterials 2018, 159, 204

[42] A. K. Gaharwar, L. M. Cross, C. W. Peak, K. Gold, J. K. Carrow, A. Brokesh, K. A. Singh, Adv. Mater. 2019, 31, 1900332.

[43] S. Basu, S. Pacelli, Y. Feng, Q. Lu, J. Wang, A. Paul, ACS Nano 2018, 12, 9866.

[44] L. Tao, L. Zhonglong, X. Ming, Y. Zezheng, L. Zhiyuan, Z. Xiaojun, W. Jinwu, RSC Adv. 2017, 7, 54100

[45] D. Su, L. Jiang, X. Chen, J. Dong, Z. Shao, ACS Appl. Mater. Interfaces 2016, 8, 9619

[46] J. R. Xavier, T. Thakur, P. Desai, M. K. Jaiswal, N. Sears, E. Cosgriff-Hernandez, R. Kaunas, A. K. Gaharwar, ACS Nano 2015, 9, 3109.

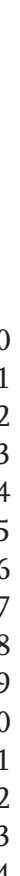


[47] C. Loebel, M. D’Este, M. Alini, M. Zenobi-Wong, D. Eglin, Carbohydr. Polym. 2015, 115, 325.

[48] L. Pickart, A. Margolina, Int. J. Mol. Sci. 2018, 19, 1987.

[49] A. Darr, A. Calabro, J. Mater. Sci.: Mater. Med. 2009, 20, 33.

[50] C. Loebel, S. E. Szczesny, B. D. Cosgrove, M. Alini, M. Zenobi-Wong, R. L. Mauck, D. Eglin, Biomacromolecules 2017, 18, 855

[51] J. Lee, M. Ju, O. H. Cho, Y. Kim, K. T. Nam, Adv. Sci. 2019, 6, 1801255.

[52] K. Xu, K. Narayanan, F. Lee, K. H. Bae, S. Gao, M. Kurisawa, Acta Biomater. 2015, 24, 159.

[53] Y. Liu, H. Meng, S. Konst, R. Sarmiento, R. Rajachar, B. P. Lee, ACS Appl. Mater. Interfaces 2014, 6, 16982.

[54] A. J. Engler, S. Sen, H. L. Sweeney, D. E. Discher, Cell 2006, 126, 677.

[55] O. Chaudhuri, L. Gu, D. Klumpers, M. Darnell, S. A. Bencherif, J. C. Weaver, N. Huebsch, H.-P. Lee, E. Lippens, G. N. Duda, D. J. Mooney, Nat. Mater. 2016, 15, 326.

[56] V. B. Bueno, R. Bentini, L. H. Catalani, D. F. S. Petri, Carbohydr. Polym. 2013, 92, 1091.

[57] S. K. Hahn, J. K. Park, T. Tomimatsu, T. Shimoboji, Int. J. Biol. Macromol. 2007, 40, 374.

[58] H.-Y. Lee, C.-H. Hwang, H.-E. Kim, S.-H. Jeong, Carbohydr. Polym 2018, 186, 290.
[59] J. A. Burdick, G. D. Prestwich, Adv. Mater. 2011, 23, H41.

[60] S. Sharifi, S. B. C. Blanquer, T. G. van Kooten, D. W. Grijpma, Acta 2 Biomater. 2012, 8, 4233

[61] M. E. Klontzas, S. Reakasame, R. Silva, J. C. F. Morais, S. Vernardis, R. J. MacFarlane, M. Heliotis, E. Tsiridis, N. Panoskaltsis, 5 A. R. Boccaccini, A. Mantalaris, Acta Biomater. 2019, 88, 224.

[62] H. Yukawa, K. Suzuki, K. Aoki, T. Arimoto, T. Yasui, N. Kaji, 6 T. Ishikawa, T. Ochiya, Y. Baba, Sci. Rep. 2018, 8, 6765.

[63] S. Jose, M. L. Hughbanks, B. Y. K. Binder, G. C. Ingavle, J. K. Leach, 8 Acta Biomater. 2014, 10, 1955.

[64] J. Pauty, R. Usuba, I. G. Cheng, L. Hespel, H. Takahashi, 10 K. Kato, M. Kobayashi, H. Nakajima, E. Lee, F. Yger, F. Soncin, 11 Y. T. Matsunaga, EBioMedicine 2018, 27, 225.

[65] X. Wang, B. Liu, Q. Xu, H. Sun, M. Shi, D. Wang, M. Guo, ]. Y C. Zhao, B. Feng, Wound Repair Regener. 2017, 25, 270. H. Nary-Filho, Clin. Oral Implants Res. 2017, 28, 320.

[67] N. R. Raia, B. P. Partlow, M. McGill, E. P. Kimmerling, C. E. Ghezzi, 16 D. L. Kaplan, Biomaterials 2017, 131, 58.

[68] A. Mata, L. Palmer, E. Tejeda-Montes, S. I. Stupp, Methods Mol. 18 Biol. 2012, 811, 39. 


\section{Reprint Order Form}

Charges for Reprints in Euro (excl. VAT), prices are subject to change. Minimum order 50 copies; single issues for authors at a reduced price.

\begin{tabular}{l|rrrrrr} 
No. of pages & $\begin{array}{c}\mathbf{5 0} \\
\text { copies }\end{array}$ & $\begin{array}{c}\mathbf{1 0 0} \\
\text { copies }\end{array}$ & $\begin{array}{c}\mathbf{1 5 0} \\
\text { copies }\end{array}$ & $\begin{array}{c}\mathbf{2 0 0} \\
\text { copies }\end{array}$ & $\begin{array}{c}\mathbf{3 0 0} \\
\text { copies }\end{array}$ & $\begin{array}{c}\mathbf{5 0 0} \\
\text { copies }\end{array}$ \\
\hline $\mathbf{1 - 4}$ & $345,-$ & $395,-$ & $425,-$ & $445,-$ & $548,-$ & $752,-$ \\
$\mathbf{5 - 8}$ & $490,-$ & $573,-$ & $608,-$ & $636,-$ & $784,-$ & $1077,-$ \\
$\mathbf{9 - 1 2}$ & $640,-$ & $739,-$ & $786,-$ & $824,-$ & $1016,-$ & $1396,-$ \\
$\mathbf{1 3 - 1 6}$ & $780,-$ & $900,-$ & $958,-$ & $1004,-$ & $1237,-$ & $1701,-$ \\
$\mathbf{1 7 - 2 0}$ & $930,-$ & $1070,-$ & $1138,-$ & $1196,-$ & $1489,-$ & $2022,-$ \\
\hline & & & & & & \\
every additional & $147,-$ & $169,-$ & $175,-$ & $188,-$ & $231,-$ & $315,-$ \\
4 pages & & & & & &
\end{tabular}

Please send me send bill me for

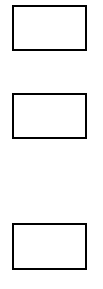

no. of reprints

no. of issue

(1 copy: 28 Euro)

high-resolution PDF file (330 Euro excl. VAT)

E-mail address:

* Special Offer:

If you order 200 or more reprints you will get a PDF file for half price.

Please note: It is not permitted to present the PDF file on the internet or on company homepages.

Cover Posters (prices excl. VAT)

Posters of published covers are available in two sizes:

$\square$ DIN A2 $42 \times 60 \mathrm{~cm} / 17$ × 24in (one copy: 39 Euro)

DIN A1 60 x 84 cm / 24 x 33in (one copy: 49 Euro)

Postage for shipping (prices excl. VAT)

overseas +25 Euro

within Europe +15 Euro
Email: afm@wiley-vch.de

Manuscript No.:

Customer No.: (if available)

Purchase Order No.:

Author:

Information regarding VAT: The charges for publication of cover pictures /reprints/issues/poster/Video abstracts/ are considered to be "supply of services" and therefore subject to German VAT. However, if you are an institutional customer outside Germany, the tax can be waived if you provide us with the valid VAT number of your company. Non-EU customers may have a VAT number starting with "EU" instead of their country code, if they are registered with the EU tax authorities. If you do not have a valid EU VAT number and you are a taxable person doing business in a non-EU country, please provide a certification from your local tax authorities confirming that you are a taxable person under local tax law. Please note that the certification must confirm that you are a taxable person and are conducting an economic activity in your country. Note: certifications confirming that you are a taxexempt legal body (non-profit organization, public body, school, political party, etc.) in your country do not exempt you from paying German VAT.

\section{VAT number:}

Mail reprints / copies of the issue to:

Send bill to:

I will pay by bank transfer

I will pay by credit card

\section{VISA, Mastercard and AMERICAN EXPRESS}

For your security please use this link (Credit Card Token Generator) to create a secure code Credit Card Token and include this number in the form instead of the credit card data. Click here: https://www.wiley-vch.de/editorial production/index.php CREDIT CARD TOKEN NUMBER 